\title{
Percepción de los efectos del cambio climático en las actividades productivas de las localidades aledañas a la cuenca hidrográfica Aguas Zarcas, Alajuela, Costa Rica
}

\author{
Josellyn Karina Ávila Murillo, Bach. ${ }^{1}$ \& Adrián Ruiz Rodríguez, M. Sc. ${ }^{2}$ \\ 1. Movimiento Culturas Vivas Comunitarias Costa Rica; josyavila77@gmail.com \\ 2. Universidad Estatal a Distancia; aruiz@uned.ac.cr
}

Recibido: 06 marzo 2016

Aceptado: 09 abril 2016

\section{RESUMEN}

Se presenta la percepción de una muestra de la comunidad de Aguas Zarcas sobre el cambio climático, sus efectos e impactos sobre las actividades vinculadas con la cuenca hidrográfica del mismo nombre. Se logró inferir que tanto productores, comerciantes agropecuarios y residentes perciben cambios en el clima, principalmente temperaturas y variación en las precipitaciones, por lo que sus actividades son afectadas y vinculadas a la incertidumbre del estado del tiempo, lo que les hace requerir alternativas que les permita adaptarse a estos cambios.

Palabras clave: Aguas Zarcas, Climate Change, hydrographic basin.

\begin{abstract}
The study took place in Aguas Zarcas, one of the most important community in the same name watershed in the Northern Region of Costa Rica. Local perceptions related to climate change and its impacts on the land use and local production are included. Farm owners, rural workers or sale workers such as local residents; had recognized climate variations, mainly in the temperature and rainfall patterns. They had mentioned uncertainty in the forecast and weather scenarios, consequently, affectation on their activities. Besides, informers had recognized the lack of adaptation strategies in their activity to face the phenomena.
\end{abstract}

Key words: Aguas Zarcas, Climate Change, watershed.

\section{Introducción}

Con el fenómeno global calentamiento global se esperan muchos cambios para el planeta, tales como: aumento de temperaturas, variación en las precipitaciones, aumento del nivel del mar y fenómenos naturales. Por ende; habrá una modificación de la biodiversidad, alteraciones del clima, aumento de plagas y enfermedades (Grupo Intergubernamental de Expertos sobre el Cambio Climático, 2002). Debido a estos cambios, Costa Rica está participando de iniciativas como ser Carbono Neutral 2021 (DDC, sf) como también cumplir con el Plan Estratégico para la Diversidad Biológica 2011-2020 y las Metas de Aichi para lograr una adaptación al cambio climático. Sin embargo estas iniciativas no son tan rápidas de ejecutar en todo el territorio nacional, por lo que en este trabajo se abordan parte de las percepciones de impactos identificadas por la comunidad que rodea la cuenca hidrográfica Aguas Zarcas, en especial del tipo agropecuario, ambiental y productivo.

El fin primordial del trabajo fue determinar los efectos e impacto del cambio climático sobre las actividades productivas que rodean la cuenca hidrográfica Aguas Zarcas en el distrito de Aguas Zarcas, San Carlos, Alajuela, Costa Rica.

\section{Área de estudio}

Aguas Zarcas se ubica en el distrito número cuatro de San Carlos con el mismo nombre. Posee una población aproximada de 20241 habitantes. Se ubica a 15 kilómetros de la cabecera del cantón Ciudad Quesada y a 489 m.s.n.m. Su cuenca hidrográfica se encuentra en una zona que presenta un área de $159,04 \mathrm{~km}^{2}$ el cual ocupa un $4,75 \%$ del área total del cantón, donde el 
crecimiento urbanístico ha aumentado en los últimos años (Municipalidad de San Carlos, 2014).

La cuenca hidrográfica Aguas Zarcas nace en las alturas del Parque Nacional Juan Castro Blanco, exactamente en la formación montañosa de los Cerros Porvenir o Congo, Platanar, Avión, El Siete y el Pelón que representan una altura máxima de 2339 m.s.n.m. (Parque Nacional del Agua, 2009). Exactamente en las faldas del norte en que se ubica la Cordillera Volcánica Central del país presentando un suelo Andosol los cuales se caracterizan por ser poco evolucionados, presentando cenizas volcánicas, son suelos pesados y de poca profundidad, además son suelos compactados (Solano \& Villalobos, sf).

El tipo de vegetación en la zona es muy variable desde el bosque muy húmedo montano en la parte más alta de la región con biotemperaturas entre $9^{\circ} \mathrm{C}$ y $12^{\circ} \mathrm{C}$, en donde el bosque se caracteriza por ser inalterado, siempre verde, dos estratos y altura poco densa. Al igual presenta un bosque muy húmedo subtropical en el que la biotemperatura puede variar entre los $17^{\circ} \mathrm{C}$ y $24^{\circ} \mathrm{C}$. Es caracterizado por ser un bosque de mediana altura, siempre verde y presentando alrededor de dos o tres estratos (Solano \& Villalobos, sf).

\section{Metodología}

Los siguientes son datos de Ávila con apoyo de Ruiz (2015):

\section{Tipo de estudio}

El trabajo presentará un enfoque de investigación mixto exploratorio que pretende interiorizar desde la perspectiva social aspectos que afectan e impactan la actividad productiva en el área de estudio por causa del cambio climático.

Este enfoque permite comprender e interpretar la realidad que enfrentan los actores sociales en estudio desde una visión más amplia (Galeano, 2004).

Se hizo un muestreo de la población del tipo probabilístico, estratificado y por conglomerados; también se recurrió al muestreo no probabilístico, en el que se utilizará el muestreo intencional u opinático. Para esa fase se obtuvieron los datos por medio de observaciones, visitas de campo, entrevistas, encuestas, toma de notas de campo y registro fotográfico.

Se utilizó además el método de evaluación diagnóstica participativa con el fin de encontrar el principal o principales problemas que enfrenta la zona de estudio en conjunto con los principales actores sociales. Este fue un tipo de evaluación diagnóstica externa y rápida para determinar las medidas y acciones que se deben tomar en cuenta para minimizar los efectos e impactos del cambio climático en las actividades productivas que rodean la cuenca del el río Aguas Zarcas.

Las técnicas y recolección de datos serán por medio de búsqueda de documentación científica y citada, observaciones, y fotografías. Además de ello; se utilizará la técnica Delphi (Delfos) que será aplicada a técnicos y especialistas que presentan conocimiento sobre el problema que enfrenta la zona de estudio.

\section{Población objeto}

Se identificaran dos subgrupos, la población afectada y la población no afectada directamente.

El primer subgrupo serán los productores agropecuarios y el sector económico que se relacione directamente con las actividades productivas de la zona, por medio de la identificación de los efectos e impactos que el cambio climático está presentando sobre las actividades productivas.

El segundo subgrupo serán los vecinos de las zonas altas y media que rodean el área de estudio con la finalidad de identificar la situación socioambiental y socio-económica que los circunda.

\section{Criterios de las unidades de estudio}

Según el INEC (2011), en su último censo el distrito de Aguas Zarcas presenta una densidad de población de 20239 personas en total, en la cual incluyen niños (as), adolescentes, adultos (as) y adultos (as) mayores. En donde se específica que el 41,4\% de la población pertenece al área urbana del territorio, siendo éste parte del territorio que será estudiado, en donde la cuenca 
hidrográfica Aguas Zarcas se sitúa tanto la parte alta como parte media de la misma.

Las personas que estarán bajo estudio deberán residir en una franja de un kilómetro de circunferencia de la cuenca hidrográfica Aguas Zarcas. Dicho kilómetro obedece a los dos transectos demarcados para realizar el estudio, debido a su importancia socio-económica y ambiental en la zona. Esta población puede ser tanto de nacionalidad costarricense como extranjeros con edades entre los 14 y 80 años que residan permanente en la zona; esto para el caso de la población no afectada.

Para la población afectada se deberá tomar en cuenta que sean productores agropecuarios y que se ubiquen en la franja anterior mencionada. Por otro lado, la selección de la población dedicada a las actividades económicas debe estar ligada a las actividades productivas y estar formal y legalmente establecidos.

\section{Fuentes de información}

La información que se utilizará vendrá tanto de fuentes primarias como secundarias. Las fuentes primarias se obtendrán por medio de entrevistas con un cuestionario previamente realizado; así como trabajo de campo, registro fotográfico y observaciones en la zona de estudio.

Por su parte, las fuentes secundarias serán basadas en información documentada: libros, registros, investigaciones previamente realizadas, informes y otros documentos.

\section{Variables y herramientas para su estudio}

Se tomaron las siguientes variables para la realización del estudio según las actividades productivas y económicas afectadas por el cambio climático de la zona:

- Cantidad estimada de productores agropecuarios.

- Cantidad estimada de actividades económicas relacionadas con la productividad del área de estudio.
- Ubicación de los pueblos cercanos a la cuenca hidrográfica Aguas Zarcas (Los Chiles, Caporal, Aguas Zarcas Centro, Calle Damas, Loma y Montecristo).

También se tomaron variables más específicas según las actividades productivas y económicas:

- Años de ejecutar las actividades económicas y productivas.

- Impacto de las actividades en la economía de la zona de estudio.

- Vínculos con instituciones gubernamentales.

En el sector medio de la cuenca está dedicado en su mayoría a la actividad comercial, en el cual se hallan las empresas que se dedican a ofrecer los suministros agropecuarios que los productores requieren en la región.

Es por esta razón que Aguas Zarcas se caracteriza por su importancia económica en el cantón de San Carlos y por el papel que juega ambientalmente al presentar fuentes de agua indispensables para el abastecimiento humano y por una parte de la zona protectora del Parque Nacional Juan Castro Blanco.

\section{Delimitación}

Para la realización del estudio se delimitará la cuenca en dos transectos de la cuenca hidrográfica Aguas Zarcas. El transecto $\mathrm{N}^{\circ} 1$ correspondió con la parte alta de la cuenca que delimita con el Parque Nacional Juan Castro Blanco y las nacientes de agua del Acueducto y Alcantarillado que abastece tanto al distrito de Aguas Zarcas y a al distrito vecino de la Palmera; como también con sectores de uso agropecuario. El transecto $\mathrm{N}^{\circ} 2$ se realizó en la parte media de la cuenca, en la que se halla la parte céntrica del distrito y algunas áreas de uso agropecuario y turístico de la zona.

Los barrios o poblados a tomar en cuenta para el estudio serán Montecristo, Calle Damas, Aguas Zarcas Centro (comercio 
agropecuario únicamente), Caporal y Los Chiles respectivamente.

\section{Resultados}

Estos datos se obtuvieron de una población muestra de 88 personas entre los 14 años hasta los 80 años que se visitaron en sus lugares de residencia y que se encontraron dispuestas a colaborar en la investigación. Lamentablemente la respuesta de la población meta fue baja debido al temor de responder la encuesta; puesto que tenía desconocimiento del tema o por razones personales no estuvieron en la disponibilidad de responder a la misma.

\section{ÍNDICE DE LAS ACTIVIDADES SOCIOECONÓMICAS A LAS QUE SE DEDICA LA POBLACIÓN MUESTRA}

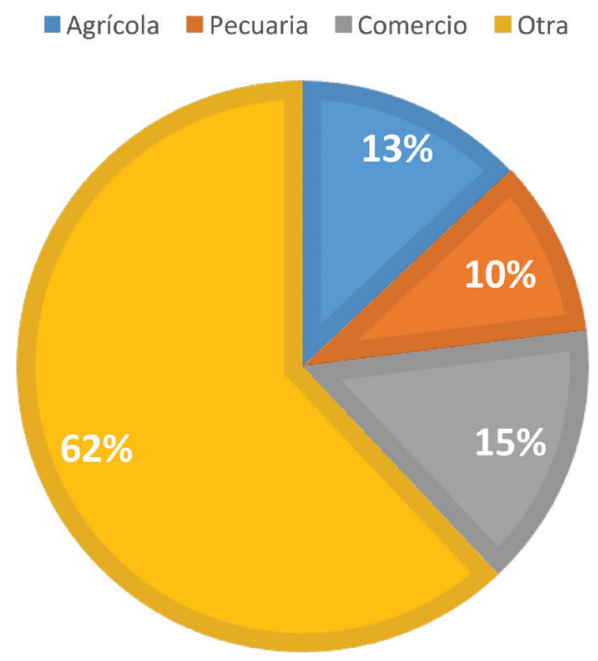

Gráfico No 1. Representación del Índice de las actividades socioeconómicas a las que se dedica la población muestra no afectada por el cambio climático en los alrededores de la cuenca Aguas Zarcas. Fuente: Elaboración propia con base en encuestas de trabajo de campo, 2015.

\section{ÍNDICE DE CONOCIMIENTO DE LA POBLACIÓN MUESTRA SOBRE EL CAMBIO CLIMÁTICO}

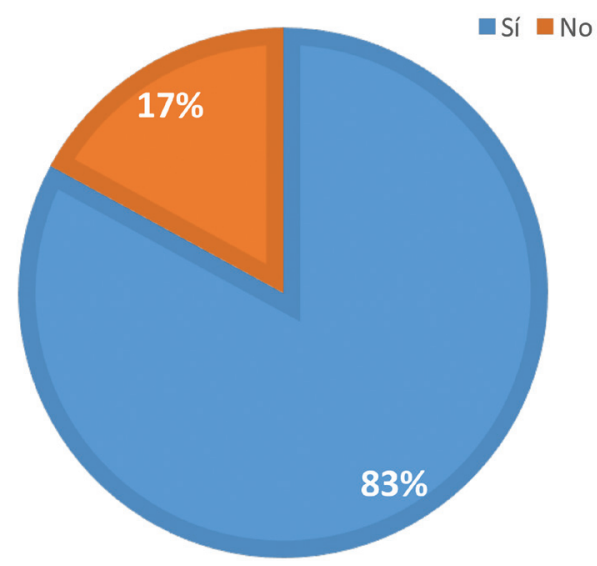

Gráfico № 2. Representación del Índice de conocimiento que presenta la población muestra sobre el tema de cambio climático. Fuente: Elaboración propia con base en encuestas de trabajo de campo, 2015.

\section{ÍNDICE DE CONOCIMIENTO DE LA POBLACIÓN MUESTRA SOBRE LOS EFECTOS E IMPACTOS DEL CAMBIO CLIMÁTICO SOBRE EL PLANETA}

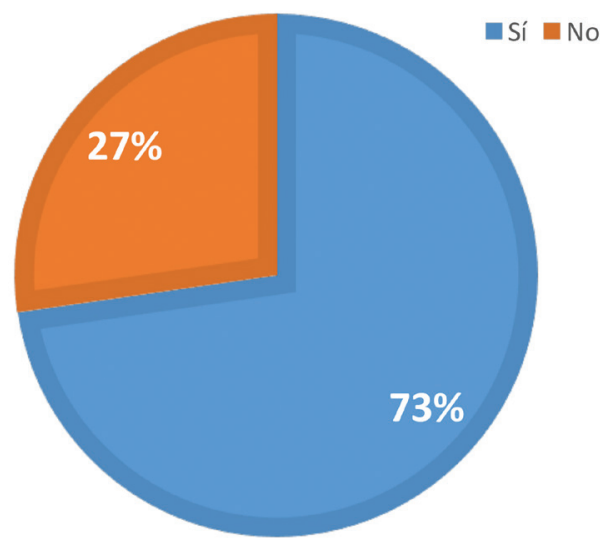

Gráfico № 3. Representación del Índice de conocimiento que presenta la población muestra sobre los efectos e impactos del cambio climático sobre el planeta. Fuente: Elaboración propia con base en encuestas de trabajo de campo, 2015. 
OPINIÓN A LA INTERROGANTE ¿ESTÁ AFECTANDO EL CAMBIO CLIMÁTICO EL PLANETA?

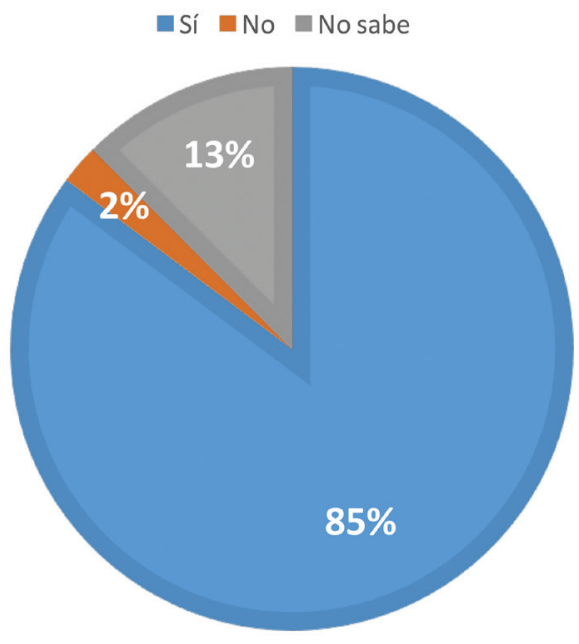

Gráfico № 4. Representación de lo que la población muestra opina a la interrogante ¿Está el cambio climático afectando negativamente el planeta? Fuente: Elaboración propia con base en encuestas de trabajo de campo, 2015.

\section{OPINIÓN A LA INTERROGANTE ¿SE VEN AFECTADAS LAS ACTIVIDADES PRODUCTIVAS Y ECONÓMICAS POR CAUSA DEL CAMBIO CLIMÁTICOS}

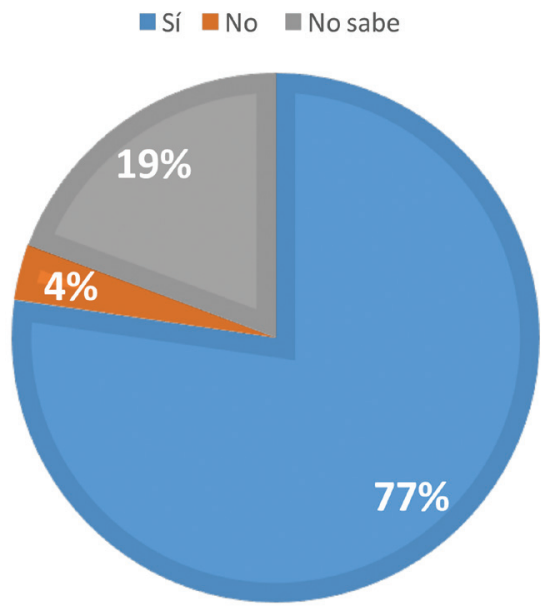

Gráfico № 5. Representación de la opinión que presenta la población muestra con respecto a la interrogante ¿Se ven afectadas las actividades productivas y económicas de la zona por causa del cambio climático? Fuente: Elaboración propia con base en encuestas de trabajo de campo, 2015.
Se obtuvieron los datos donde se específica por qué el cambio climático está afectando las actividades productivas y económicas que rodean la cuenca hidrográfica Aguas Zarcas, habiendo resultados similares en la mayoría de respuestas. Las personas que consideraron que sí está afectando (77\% de la población) concluyeron en que las variaciones de clima se evidencias en los incrementos de temperatura, bajas precipitaciones en algunas épocas, con un contraste en las que en otros espacios temporales las precipitaciones son muy abundantes.

Destacan como causas la deforestación, problemas en el suelo y contaminación ambiental. En contraste, una minoría del $4 \%$ que opinó que estos factores no afectan a las actividades productivas y económicas de la zona en estudio.

\section{OPINIÓN A LA INTERROGANTE ¿HA AUMENTADO EL CAMBIO CLIMÁTICO EN LA ZONA?}

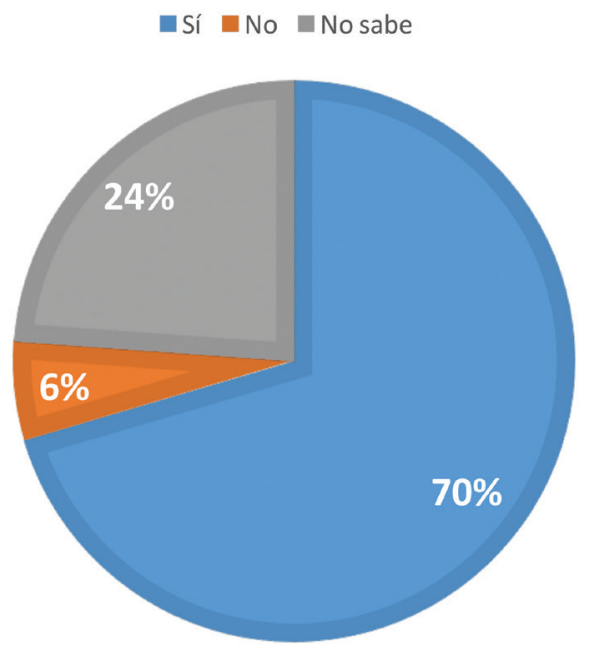

Gráfico № 6. Representación de la opinión de la población muestra que rodea la cuenca hidrográfica Aguas Zarcas hacia la interrogante $¿ \mathrm{Ha}$ aumentado el cambio climático en la zona? Fuente: Elaboración propia con base en encuestas de trabajo de campo, 2015. 
En preguntas de comprobación, llamó mucho la atención la reiteración de datos: El 70\% de la población consideró que el cambio climático se da debido a que ha habido una variación climática en el incremento de la temperatura promedio y descontrol en la cantidad de precipitaciones anuales. Afirman que en algunas épocas llueve más y en otras la lluvia no es tan abundante como hace algunos años atrás. De nuevo, una breve frecuencia ligeramente mayor (6\%), opina que el clima de la zona sigue igual o no ha sufrido un cambio tan drástico, por lo que opinan que el cambio climático no ha aumentado en el área.

\section{ÍNDICE DE CONOCIMIENTO DE LA POBLACIÓN SOBRE LA EXISTENCIA DE INSTITUCIONES QUE TRABAJEN PARA MINIMIZAR LOS EFECTOS E IMAPACTOS DEL CAMBIO CLIMÁTICO SOBRE LAS ACTIVIDAD PRODUCTIVAS DE LA ZONA}

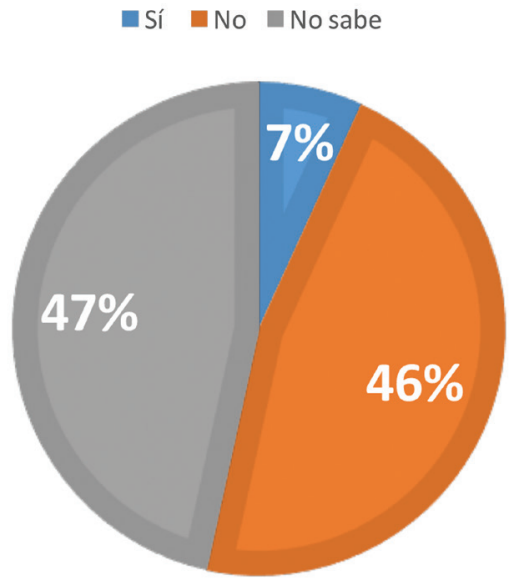

Gráfico № 7. Representación del índice de conocimiento que presenta la población muestra con respecto a las instituciones gubernamentales que están trabajando en la comunidad para minimizar efectos e impactos que el cambio climático está ejerciendo sobre las actividades productivas. Fuente: Elaboración propia con base en encuestas de trabajo de campo, 2015.

Se encontraron resultados de opinión muy bajos con respecto a la existencia de instituciones gubernamentales enfocadas en minimizar el cambio climático sobre las actividades productivas de la zona. Un dato muy interesante es que las instituciones indicadas por la población que se encuentran trabajando por minimizar los efectos e impactos del cambio climático no son necesariamente instituciones del gobierno dedicadas al sector ambiental o agropecuario. Las instituciones mencionadas fueron:

- El Ministerio de Educación Pública trabajando en el reciclaje y reforestación.

- La Seguridad Comunitaria realizando charlas educativas sobre el cambio climático y cómo minimizar los efectos e impactos que provoca.

- La Municipalidad de San Carlos realizando campañas en pro del ambiente y;

- El Ministerio de Salud está trabajando para mitigar las quemas ambientales.

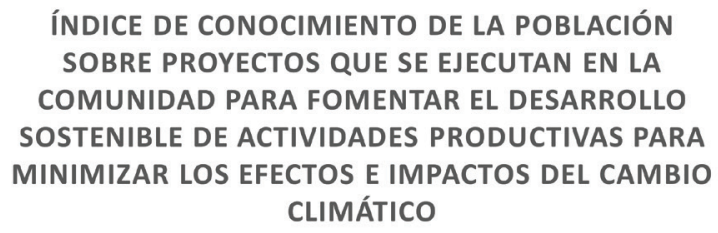

- Si existen $\quad$ No existen $\quad$ Desconoce

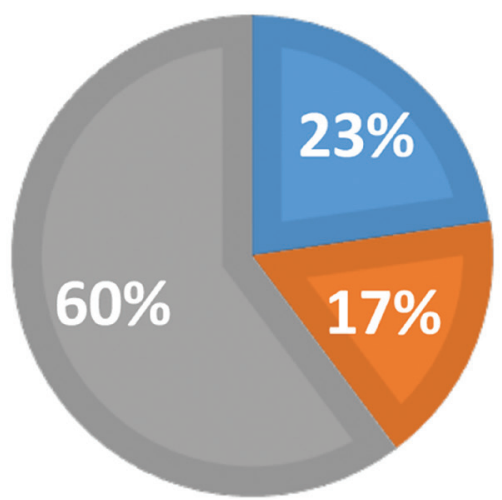

Gráfico № 8. Representación de la población muestra que conoce sobre proyectos que se ejecutan con la finalidad de promover el desarrollo sostenible de las actividades productivas que permitan minimizar los efectos e impactos del cambio climático en la zona que rodea la cuenca hidrológica de Aguas Zarcas. Fuente: Elaboración propia con base en encuestas de trabajo de campo, 2015.

Algunos de los proyectos e instituciones que los están ejecutando son los siguientes: agricultura orgánica que pretende fomentar el desarrollo agroecológico por parte del Colegio Técnico Profesional Nataniel Arias Murillo, reforestación por parte de varias instituciones (Coocique R.L, La Hora Ecológica, ICE, Municipalidad de 
San Carlos, Coopelesca, Escuela Mario Salazar, Acueductos y Alcantarillados, y Vecinos de las comunidades), reciclaje por parte de escuelas y vecinos.

También se realiza monitoreo preventivo contra la deforestación por parte del Ministerio del Ambiente y Energía (MINAE), limpieza de ríos y zonas aledañas por parte de la Municipalidad de San Carlos, programa de bandera azul por parte de Acueductos y alcantarillados (AYA) y limpieza de desagües por parte de la asociación de desarrollo para proteger los ríos.
Sin embargo, una parte de la población muestra afirmó que en la zona no se realiza ningún tipo de proyecto que fomente el desarrollo sostenible y la mitigación del cambio climático.

Según el gráfico $\mathrm{N}^{\circ} 9$ el promedio que la población muestra le da a las instituciones según las acciones que se encuentran realizando para controlar los efectos e impactos del cambio climático es de 3 puntos que equivale a un trabajo regular por parte de estas autoridades gubernamentales.

\section{Calificaciones dadas por parte de la población muestra a cada institución según sus acciones para controlar los efectos e impactos del cambio climático}

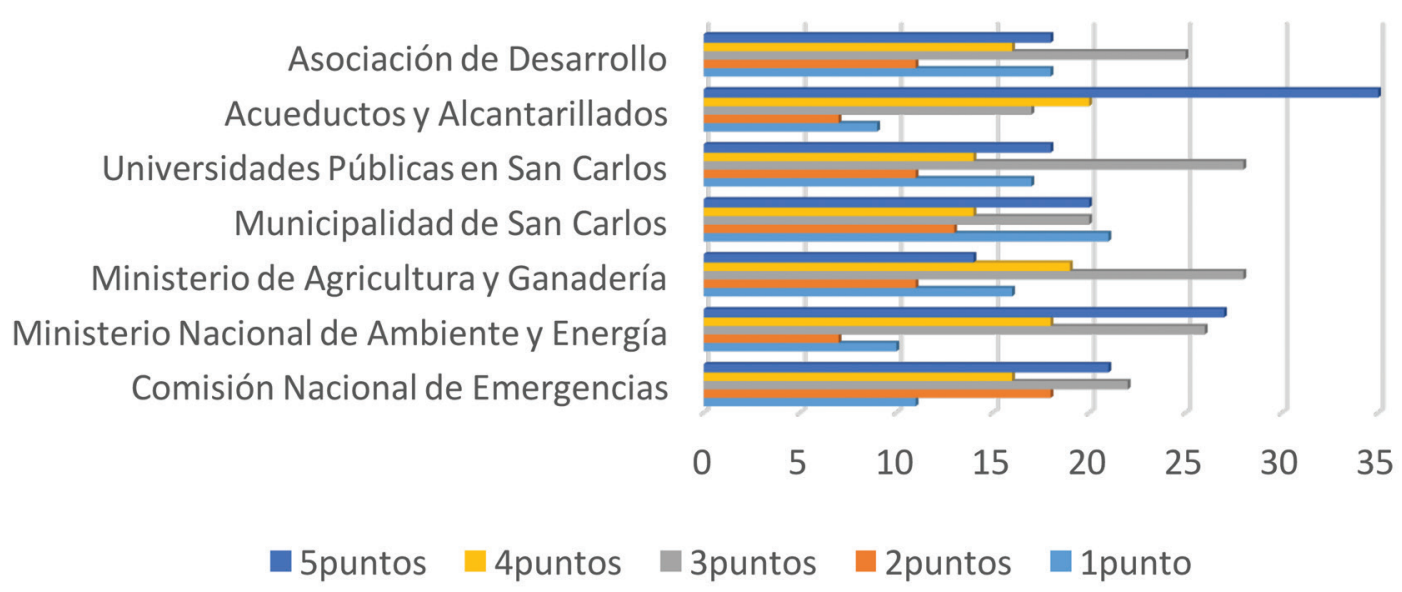

Gráfico № 9. Representación de las calificaciones dadas por parte de la población muestra a cada institución según los esfuerzos que están realizando para controlar los efectos e impactos del cambio climático en la comunidad. Fuente: Elaboración propia con base en encuestas de trabajo de campo, 2015.

\section{Segunda parte}

En esta sección se obtuvieron los resultados de la segunda población muestra afectada por el cambio climático que rodea a la cuenca hidrográfica Aguas Zarcas. Se entrevistaron tanto productores como comerciantes agropecuarios de la zona para obtener la información requerida para demostrar los problemas que enfrenta esta población por causa del cambio climático.

\section{Resultados de los comercios agropecuarios}

\section{Pro Agro S.A}

Según Eladio Camacho, propietario de la empresa Pro Agro S.A la cual se dedica a ofrecer suministros agropecuarios desde hace 28 años menciona que los comercios se están viendo afectados indirectamente por el cambio 
climático debido a la variabilidad climática en épocas lluviosas y secas, lo que implica que las programaciones agrícolas no se logren realizar de manera eficiente, afectando a los comercios debido a que los productores minimizan las compras de insumos agrícolas que la empresa ofrece.

Menciona que su establecimiento ha tenido bajas económicas en los últimos 5 años porque muchos de sus clientes han dejado de producir cultivos o en otros casos el área de siembra se reduce. También menciona que las instituciones gubernamentales aún no se han acercado a su empresa a ofrecer ayuda con respecto a cómo sobrellevar el tema del cambio climático en las actividades económicas. Además considera importante que estas instituciones informen a la población en general de los cambios climáticos y qué medidas se deben tomar y cómo prevenirlos y su vez, cree que se debería capacitar a toda la población para mitigar de una u otra forma.

Por otro lado el señor Camacho no logró dar soluciones para mitigar los efectos e impactos del cambio climático sobre las actividades productivas y económicas de la zona porque cree que primero debe estar capacitado e informado antes de buscar soluciones para minimizar el cambio climático. Mencionó que la cuenca Aguas Zarcas ha bajado sus niveles de agua y tierra por lo que considera que en un futuro las zonas aledañas a la misma serán vulnerables frente al cambio climático. Sin embargo, considera que al nacer la cuenca del Parque Nacional Juan Castro Blanco un área protegida no se encuentra afectada, lo mismo sobre la producción de energía eléctrica que se genera sobre el embalse de la cuenca, pues se ha visto afectada por el cambio climático debido a que el nivel del agua ha bajado.

Por último, el propietario del comercio mencionó que él cree necesario un plan regulador o de manejo que mitigue los efectos e impactos del cambio climático sobre las actividades productivas; como también ha considerado realizar un plan de manejo en su empresa, pero no ha sido concretado por falta de información que le permita guiarse.

\section{Empresa Agropecuaria Dos Pinos}

La Ing. Agrónoma Kathia Abarca de la empresa Agropecuaria Dos Pinos ubicada en Aguas Zarcas, mencionó que también se ven afectados debido a que el principal objetivo de la empresa son los pastos que se producen en la zona para la actividad lechera y al percibir alteraciones en el clima deben buscar nuevas forma de adaptación incrementando los costos, ya que deben controlar la plagas, realizar cruces genéticos más resistentes al cambio y que los productos logren controlar nuevas enfermedades en los pastos. Sin embargo mencionó que la empresa no ha presentado bajas económicas, pero que ha sido más difícil cumplir con los objetivos de venta debido a que el productor tiene menor capacidad de compra. También destacó que la propia empresa realiza capacitaciones sobre el cambio climático y cómo enfrentarlo tanto a los empleados como a los productores; al igual señaló que el Gobierno de Costa Rica no está apoyando a los comercios agropecuarios y cree que es indispensable que apoyen y realicen capacitaciones o charlas, pero es necesario que el sistema sea eficiente y transparente para lograrlo.

Entre las soluciones dadas por la Ingeniera se encuentra la irrigación, establecimiento de pasturas, ayudar a los productores para que logren obtener el alimento que requieren para sus animales, captación de agua de lluvia por medio de tanques y la protección de las nacientes.

Considera que la productividad agropecuaria de la zona si se ve afectada por el cambio climático, pero no como en otras regiones del país debido a que se encuentra el Parque Nacional Juan Castro Blanco cerca de la cuenca; sin embargo las plagas han aumentado por causa de un incremento de la temperatura y la humedad. También menciona que el cambio climático está afectando el caudal de la cuenca, ya que éste ha disminuido. Afectando también las actividades productivas, ya que en ciertas zonas no se puede captar agua y la contaminación generada no es apta para el consumo productivo. 
Finalizando, la Ing. Abarca apunta que es importante un plan regulador que mitigue los impactos y efectos del cambio climático sobre las actividades productivas debido a que el riesgo económico y ambiental minimizaría; por ende, el beneficio social se incrementaría. Menciona que la empresa si presenta un plan de manejo en el cual se abarcan charlas a los productores y se están entregando financiamientos para que los productores logren adaptarse al cambio climático y con ello minimizar el impacto en sus actividades.

\section{Resultados de los productores}

La finca productora de leche

de don Eddie Arrieta

El señor Arrieta afirmó que tiene aproximadamente 12 años de trabajar y según nos menciona han tenido que tomar medidas como lo es estabular el ganado lechero para que no se vea afectado por el incremento de temperaturas y precipitaciones lo cual ha bajado la producción de leche.

Mencionó que si han recibido charlas e información sobre el cambio climático por parte de empresas privadas (Dos Pinos y SIGMA), sin embargo cree que es importante que el MAG los asesore mejor, preocuparse más por informar y orientar a los productores en especial a los que no tienen acceso a la información.

Además de que este tipo de información, charlas o talleres que se ofrezcan ayudaría a prevenir problemas por causa del cambio climático en la zona, especialmente en el sector ganadero y agrícola. Por su parte don Eddie trata de evitar quemas ambientales y deforestación como también dar un adecuado manejo de residuos sólidos producidos en la finca.

El productor señala que si la población no hace conciencia sobre el daño ambiental que se está haciendo el cambio climático va a afectar más a la cuenca debido a que las actividades productivas se están acercando al Parque Nacional Juan Castro Blanco sitio de donde la cuenca nace, por lo que puede llegar a formarse un problema mayor, ya que el caudal se ha reducido en los últimos años. Sin embargo, la finca no se ha visto afectada por el cambio que la cuenca ha tenido en su caudal debido a que no utilizan el agua proveniente del río en sus actividades productivas, pero la actividad que sí ha sido afectada es la generación de energía hidroeléctrica en la zona y la población de la cuenca baja.

Finalmente explica que es importante realizar un plan regulador en la zona que es muy productiva para mitigar los efectos del cambio climático. También Mencionó que en particular en su finca no han pensado en un plan específico, pero si han tratado de adecuar la actividad productiva y realizar abonos orgánicos producidos por el mismo ganado para minimizar los efectos del cambio climático.

\section{Finca Las Cataratas}

Don Carlos Lobo indica que la actividad lechera a la que se dedica desde hace 10 años ha bajado la producción, ha tenido problemas en los forrajes y pastos, la dieta de los animales se ve afectada como también la preñez de sus vacas lecheras debido a la variabilidad climática en el incremento de temperatura y precipitaciones afectando toda la producción. Por lo que han tenido que variar la alimentación, el pastoreo lo cambiaron, aplicar ventilación y usar más el recurso hídrico. En donde no han tenido suficiente colaboración por parte del Ministerio de Agricultura y Ganadería (MAG) y otras entidades del Gobierno; sintiendo que es de suma importancia la ayuda que puedan ofrecer porque entre más información y prevención haya menor será el impacto en las actividades productivas por los efectos e impactos del cambio climático, señalando que con charlas, capacitaciones y talleres se lograría mitigar el impacto tanto ambiental como económico de la zona.

Algunas soluciones que menciona el señor Lobo es el reciclaje, manejo de los residuos y un manejo más organizado de las fincas productivas para dañar menos el ambiente.

Con respecto a la zona productiva mencionó que se verá afectada por el cambio climático por ser la cuenca la que genera y abastece el agua a las comunidades aledañas y que lamentablemente 
en los últimos años el nivel de su caudal se ha reducido, afectando al ganado principalmente por la escases de agua, lo que provoca que la producción no incremente.

Por último, anotó que es muy indispensable un plan regulador para las actividades productivas, ya que muchas de ellas se han establecido en sitios que no les corresponde causando graves daños como lo es la deforestación y daños al ambiente ubicadas en las cercanías del Parque Nacional Juan Castro Blanco donde nace la cuenca Aguas Zarcas. Menciona que ellos si está trabajando con un plan de manejo utilizando menos el agua potable, ya que la han cambiado por el agua de lluvia y realización de abonos orgánicos.

\section{Granja Avícola Bahía Baltry}

El señor Julio Chacón señaló que en dicha granja tienen 7 años de experiencia productiva. Mencionó que la empresa ha tenido que cambiar y realizar pruebas en la producción, ya que si la temperatura aumenta se afecta la productividad, por lo que se debe ventilar generando gastos económicos extra para la empresa debido al impacto del cambio climático en la zona. Señala que han tenido colaboración y capacitación por parte de PIPASA S.A, Servicio Nacional de Sanidad Animal (SENASA) y MAG para minimizar los efectos e impactos del cambio climático, por lo que cree que no es necesario mayor apoyo de las entidades gubernamentales. Hizo la salvedad que solo con PIPASA se ha abordado el cambio climático y que el MAG ese tema no lo ha trabajado con ellos más que en temas como control y salud animal.

Con respecto a las posibles soluciones el señor Chacón no supo dar respuesta por escasa información. Sin embargo si destaca que la zona de producción sí se ha visto afectada por el aumento de la temperatura y por ende incrementa los costos económicos para que la granja se adecue y adapte al cambio climático. Por su parte cree que el río sigue siendo el mismo caudal por lo que no se ha afectado tanto por el cambio climático y que ellos no se han visto afectados por la cuenca.
Por último, cree necesario un plan regulador para un mejor manejo de las actividades productivas y con ello obtener mejores resultados. Anotando que la granja está trabajando con un plan de manejo y que el MAG les ha solicitado un plan de desechos orgánicos e inorgánicos.

\section{Tilapias Soto}

Se visitó la finca Tilapias Soto dedicada a la acuicultura desde hace 17 años y se habló con el señor Jorge Soto. Él indicó que la temperatura ha aumentado un grado centígrado por lo que ha afectado a sus peces especialmente porque ha incrementado la cantidad de hongos en el agua resultando un grave problema para la producción. En su finca han tenido que utilizar más productos químicos para combatir los hongos y deben estar moviendo las tilapias de lugar constantemente por que las temperaturas son muy inestables, afectando el apetito de los peces debido al cambio climático. Además, han tenido que adaptar la finca para mitigar estos efectos realizando desdobles antes de las 10:00 am para que los peces no sufran estrés por las altas temperaturas y modificar el horario de alimentación solamente por las mañanas.

El señor Soto mencionó que han recibido colaboración por parte del Instituto Nacional de Aprendizaje (INA) y el MAG desde el 2002 sobe el cambio climático y cómo prevenirlo. Sin embargo dice que estas charlas no volvieron a realizarse ahora que se perciben cambios en el clima por lo que cree necesario se vuelvan a implementar. Y cree que el MAG debe de apoyar más a los productores realizando visitas antes de una crisis del cambio climático debido a que generalmente actúan cuando la crisis ya se está sufriendo.

Algunas soluciones que da para la producción acuícola para enfrentar el cambio climático es tratar de que el productor mantenga la misma cantidad de especies por metro cúbico.

Mencionó que el fenómeno de la temporada seca ha afectado las actividades productivas secando el cauce del río. Además la inestabilidad climática ha perjudicado, estando vulnerable debido a que el nivel de su caudal se reduce 
cada vez más. Sin embargo ellos no han tenido problemas directamente por la cuenca, ya que no toman agua de la misma.

Para finalizar anota que es muy importante que las organizaciones políticas y económicas tomen consciencia y poder en el Gobierno para minimizar los efectos e impactos del cambio climático ya que la deforestación desmedida está acabando con la cuenca por lo que deben de mitigar estos problemas por medio de un plan regulador. Menciona que ellos sí presentan un plan de manejo tratando de trabajar únicamente con cosecha de peces en época seca y aplicación de alimento medicado para mitigar los hongos y proliferación de bacterias durante la época de transición.

\section{Consulta a expertos institucionales}

En esta parte se encuentran los resultados obtenidos por parte de las Instituciones Gubernamentales ubicadas en las cercanías de la cuenca hidrológica Aguas Zarcas. Se localizaron las siguientes instituciones: MAG, MINAE, AYA y la Municipalidad de San Carlos para realizar una entrevista a cada una de las parte, para obtener versiones claras sobre la situación que enfrenta la comunidad con respecto al cambio climático en el sector productivo. Lamentablemente algunas de las instituciones no colaboraron con la entrevista solicitada para una mejor percepción del tema en estudio en los alrededores de la cuenca hidrológica de Aguas Zarcas.

Estas instituciones fueron AYA y MINAE las cuales solicitaron que la entrevista se realizara por vía de correo electrónico, pero fueron casos de no respuesta. Sin embargo por medio de una llamada telefónica un funcionario del MINAE mencionó que no tenía conocimiento sobre el tema de cambio climático en la zona y otro dijo que ellos eran ingenieros forestales, por lo tanto no les correspondía ese tema. Por parte del Acueducto y Alcantarillado (AYA) la respuesta fue completamente nula.

El funcionario del MAG en Aguas Zarcas José Ángel Pérez Sánchez respondió vía correo electrónico a la entrevista realizada. En el siguiente cuadro se encuentran sus respuestas al tema del cambio climático sobre las actividades productivas que rodea a la cuenca hidrológica de Aguas Zarcas y cuál ha sido el papel de Ministerio de Agricultura y Ganadería en este tema. (Cuadro 1)

\section{Cuadro No 1}

\section{Respuestas del MAG sobre el cambio climático, sus efectos e impactos en las actividades productivas que rodean la cuenca hidrológica Aguas Zarcas y su función como Institución Gubernamental. (Ver anexo 3 con las preguntas realizadas)}

\footnotetext{
"La cuenca Hidrográfica de Aguas Zarcas, comprende, parte del distrito de Aguas Zarcas y Parte del distrito de la Palmera, la causa principal es el calentamiento global, causado por la cantidad de gases que se emiten a la atmósfera (vehículos, industrias, En la actividad agropecuaria, los animales especialmente el ganado vacuno, mal uso de los agroquímicos, la quema de rastrojos."
}

"Altas precipitaciones de lluvia, afectan caminos destruyen puentes como el del río Caño Negro en Vasconia, inundan centros de población. En la actividad agropecuaria, incremento de plagas como la mosca del establo, que se reproduce en rastrojos vegetal, especialmente piña y afecta a la ganadería con picaduras."

"Las medidas preventivas ha sido capacitación a productores, sobre los efectos del clima, implementación de técnicas en mejoramiento de la alimentación animal, con pastos mejorados, división de potreros, análisis de suelo para hacer planes de fertilización, seguimiento a las fincas piñeras para que hagan un buen manejo del rastrojo." "Se apoya a las ASADAS en programas de adquisición de fincas, siembre de árboles para proteger las fuentes de agua, obras de conservación de suelo para evitar la sedimentación de ríos"

"La desforestación para siembra de monocultivo, contribuye, la ganadería por ser rumiante libera gases a la atmósfera, el uso de maquinaria agrícola con la quema de combustible, quema con fuego de rastrojo como caña de azúcar y piñales."

"La utilización de cercas vivas en vez de poste muerto, especialmente en fincas ganaderas. La mayoría de pequeños productores han cambiado la producción de piña por yuca y ganadería." 
"En Aguas Zarcas y la palmera participaron cerca de 100 productores en este tipo de charlas presencial y se va continuar, también se cuenta con programa de TV Guía Agropecuaria en canal 14 donde se informa del problema."

"El plan regulador distrital, está en proceso, se espera que para el 2016 se apruebe."

"Es posible, pero es una tarea de instituciones, fuerzas vivas gobiernos locales. Se están dando acciones aisladas."

"Varios científicos dicen que los efectos que tenemos el día de hoy, es la consecuencia de lo que se hizo hace 70 años. Todo lo que hagamos en este momento tienen resultados hasta dentro de 70 años. Pero se debe hacer cambios importantes como reciclaje, minimizar el uso de combustibles fósiles, paneles solares. dentro de las áreas agropecuarias diversificar con arboledas en bloques o en filas, seleccionar las vacas más productivas, porque es mejor tener 8 vacas excelentes que 12 de baja productividad, hacer un buen manejo d e los desechos orgánicos, el gobierno gire incentivos para la las fincas bandera azul o carbono neutralidad."

Entrevista realizada al Lic. José Ángel Pérez Sánchez, funcionario de la Agencia de Extensión Agropecuaria del MAG en Aguas Zarcas. Fuente: Elaboración propia con base en entrevista de trabajo de campo, 2015.

Por otra parte el funcionario de la Municipalidad de San Carlos, Carlos Andrei Salas Ramírez geógrafo y encargado de gestión ambiental en la municipalidad no respondió específicamente las respuestas; sin embargo aportó una presentación de power point con información muy valiosa para la investigación realizada en la cuenca hidrológica de Aguas Zarcas.

En el siguiente cuadro se aprecia un poco de lo que la municipalidad está realizando en la zona en general para minimizar los efectos e impactos del cambio climático. (Cuadro 2)

\section{CUADRO No 2}

\section{Ejes de trabajo realizados por parte de la Municipalidad de San Carlos para combatir el cambio climático en la región.}

\section{Ejes de trabajo}

Educación ambiental

Sector forestal

Sector hídrico

Sector energético

Relleno sanitario

Sector industria y uso de suelo

\section{Temas de trabajo}

Municipalidad, INA y Ministerio de Salud junto con otros actores sociales trabajan el tema de residuos sólidos y el tema de cambio climático. Protección y restauración de bosques, conservación de agua. UTN trabaja en abastecimiento, calidad, disponibilidad y corrientes de ríos y aguas subterráneas. MINAE en zonas de recarga. Trabajos para minimizar el gasto energético con las capacitaciones realizadas. Se están realizando obras de adecuación.

Están trabajando en la actualización del plan regulador, en temas de riesgo, adaptación y fragilidad ambiental.

Fuente: Salas, C. 2015. Fuente: Elaboración propia con base en entrevista de trabajo de campo, 2015.

\section{Situación diagnóstica in situ}

En esta parte se mostrarán los resultados obtenidos por medio de las observaciones y trabajo de campo realizado.

\section{Conclusiones}

En los primeros resultados obtenidos se refleja que la primera población muestra responde haber escuchado hablar sobre el tema del 


\section{CUADRO No 3}

Observaciones de campo durante las visitas a la zona de estudio a las poblaciones muestra.

Poblaciones observadas

Cuenca hidrológica de Aguas Zarcas
Detalles y resultados encontrados

Nivel del agua y el caudal bajo

Rodeado de área boscosa

Temperaturas muy elevadas para la altitud, tipo de clima y bosque presentado

Ubicado en las laderas del Parque Nacional Juan Castro Blanco

Industrias automotrices muy cerca de la cuenca

Población muestra no afectada (vecinos de la comunidad que rodean la cuenca Aguas Zarcas)

\section{Desinformación del cambio climático}

Conocen solo lo que han escuchado en los medios de comunicación Desinformación sobre proyectos relacionados con el cambio climático Poca información de los efectos e impactos del cambio climático y como mitigarlos.

Desconocimiento de las instituciones públicas que trabajan en el tema en estudio.

Población muestra afectada (productores y comerciantes agropecuarios)

Desinformación parcial sobre cómo mitigar los efectos e impactos del cambio climático en las actividades productivas

Deseosos por ser capacitados por medio de charlas, talleres $u$ otra forma de apoyo para mejorar sus prácticas agropecuarias para no repercutir en el ambiente y no afectar la economía

Requieren de un plan de manejo adecuado de sus actividades productivas Requieren mayor apoyo por parte de las instituciones gubernamentales para mitigar o prevenir el cambio climático.

No se ven afectados directamente por el bajo caudal de la cuenca, sin embargo se encuentran preocupados por ésta situación, ya que afirman que la cuenca es una de las principales fuentes de energía hidroeléctrica de la comunidad

Necesidad de un plan regulador por parte del Gobierno para prevenir y mitigar el aceleramiento del cambio climático en la región

Instituciones gubernamentales

Desinformación por parte de algunos funcionarios públicos sobe el tema Esfuerzos muy bajos para minimizar los efectos e impactos del cambio climático en la zona de estudio

Seguimiento insuficiente a los productores sobre como minimizar los efectos e impactos del cambio climático en la zona

Poca colaboración de ciertas instituciones para evacuar dudas sobre el cambio climático en los alrededores de la cuenca hidrológica Aguas Zarcas

Fuente: Elaboración propia con base en entrevista de trabajo de campo, 2015.

cambio climático, sin embargo no saben exactamente a qué se refiere este fenómeno que está afectando al planeta, ni cómo lo está impactando específicamente, presentando únicamente una leve percepción sobre cambios en temperaturas y precipitaciones en el clima, mencionando que afecta tanto a las actividades productivas como a la cuenca.

Según Elbers (2012), es común encontrar este tipo de resultados en la población mundial, la cual se vuelve una situación alarmante, ya que los seres humanos desconocen y niegan la existencia del cambio climático sobre el planeta y que la población es el principal causante de que este fenómeno aumente cada día.

Con respecto al conocimiento de trabajos y proyectos específicos ejecutados por las instituciones gubernamentales en los alrededores de la cuenca hidrográfica Aguas Zarcas para minimizar los efectos e impactos del cambio climático 
en la actividad productiva la tasa de respuesta por parte de la población fue muy baja. Por lo que demuestra que existe un proceso de comunicación e información entre Instituciones-Poblaciones sobre temas relacionados con el cambio climático bajo. Los mismos resultaros se obtuvieron al indagar si alguna organización, institución, etc. está fomentando el desarrollo sostenible en la cuenca por medio de algún proyecto que minimice los efectos e impactos del cambio climático sobre las actividades productivas.

Por otra parte la calificación dada a las instituciones gubernamentales con respecto a sus funciones para la mitigación del cambio climático en la zona fue regular, ya que los vecinos consideran que no están realizando suficientes esfuerzos por minimizar estos efectos en las cercanías de la cuenca.

Lamentablemente estos hechos se dan porque en Costa Rica tanto los actores sociales, los políticos y sociedad civil carecen de la información, poca voluntad para incluir estos temas dentro de un marco político e información poco accesible a la ciudadanía para enfrentar el cambio climático y tomar medidas de mitigación en conjunto concretas (Granados \& Madrigal, 2014).

Por otra, los resultados demuestran que los sectores dedicados al comercio agropecuario están percibiendo indirectamente impactos económicos por causa del cambio climático en las actividades productivas, debido a que la agricultura y ganadería ha disminuido en la zona; por ende una baja en el consumo de suministros agropecuarios, los cuales son ofrecidos por este tipo de mercado.

Por lo que esta población muestreada está tratando de sobrellevar estos cambios y tratando de adaptarse al impacto que está afectando a la región altamente productiva. Sin embargo se siente una preocupación por parte de esta población con respecto a cómo pueden desarrollar mecanismos de mitigación tanto en las actividades productivas como en las económicas, ya que es un tema que está perjudicando a todos tanto de manera directa como indirecta. Además de que ven la necesidad de buscar apoyo por parte de las instituciones gubernamentales que permita orientar el sector económico en temas y mecanismos sobre el cambio climático que puedan ser aplicables.

Por su parte los productores también han reflejado su preocupación por el cambio climático y las repercusiones que sus actividades productivas están presentando, ya que mencionan que han tenido que realizar inversiones para lograr adaptar sus fincas ante este hecho afectando su economía; además, las prácticas agrícolas y pecuarias han presentado problemas con respecto al cambio climático debido a las variaciones climáticas en temperaturas y precipitaciones específicamente. Esto ha provocado que la calidad de sus productos bajen o existan pérdidas tanto de materia prima como económica.

En Costa Rica las actividades agropecuarias son muy valiosas para la economía nacional, ya que estas brindan un porcentaje aproximado del $10 \%$ del PIB, sin embargo estas actividades productivas se están comenzando a ver afectadas por los efectos e impactos del cambio climático, específicamente por las variaciones del clima (las temperatura y precipitaciones) que han ocurrido en los últimos años (Ordaz \& ét. al., 2010).

El recurso hídrico es indispensable para la vida humana y por ende para las actividades productivas de una región; sin embargo es uno de los recursos más vulnerables frente al cambio climático debido a que está disminuyendo. Sin embargo los impactos de este recurso dependerán de diversos factores en la región que se encuentre, ya sea por la capacidad de abastecimiento a la población o las condiciones económicas, sociales y ambientales en las que se rodee (Watson \& ét.al, 1997). Por lo que las actividades productivas se verán afectadas si alguna de estas variables no sostiene la demanda de recurso hídrico en el sector agropecuario.

La población muestra han demostrado su interés y necesidad de buscar medidas de mitigación que permitan llevar a cabo de manera sostenible con el ambiente sus actividades agropecuarias; además de que mencionas la importancia de que las autoridades gubernamentales ejecuten planes reguladores para ser implementados en las regiones productivas como es el caso de Aguas Zarcas. 
Debido a que es una zona con muchos recursos naturales como el Parque Nacional Juan Castro Blanco y las nacientes de agua que abastecen la comunidad y que provienen de la cuenca que a su vez es indispensable para la energía eléctrica de la zona, y las cuales se encuentran vulnerables ante el cambio climático y las malas prácticas agropecuarias que se puedan generar.

Un plan regulador permite una planificación adecuado a nivel local en donde se delimitan mapas, reglas o pautas a seguir, políticas para fomentar el desarrollo y distribución de la población en cuanto a usos de tierra, conservación, áreas urbanas entre otros y requiere de un tiempo prudente para ser realizado con especialistas. Sin embargo para logar realizar un plan regulador agropecuario primero se deben tomar en cuenta aspectos como el uso de suelo de la localidad, las actividades productivas y las áreas silvestres protegidas que puedan ser vulnerables. Con esta información es posible realizar un análisis del sector y con ello ejecutar un plan regulador en la zona (Zumbado, 2011).

Por su parte, las instituciones gubernamentales como ya se mencionó anteriormente están realizando sus esfuerzos por mitigar los impactos del cambio climático tanto en las actividades productivas como en la región en general, sin embargo estos esfuerzos no han sido suficientes para controlar, orientar e informar a la población.

Por último, por medio de la información recolectada y la percepción de la población muestra se obtuvieron los datos que enlistan medidas de adaptación y mitigación en los diferentes sectores ante el cambio climático que pueden favorecer al sector socioeconómico y socioambiental de la comunidad que rodea la cuenca hidrográfica Aguas Zarcas. Sin embargo, según la Organización de las Naciones para la Agricultura y la Alimentación, la mitigación y adaptación al cambio climático no se puede realizar por sí sola, por lo que primero es necesario trabajar en conjunto para lograr reflejar los esfuerzos realizados en la región.

La población que rodea la cuenca se encuentra levemente informada y consciente sobre la situación que está enfrentando el planeta con respecto al cambio climático, no obstante requieren de una mayor orientación que les permita brindar información y evacuar dudas sobre el cambio climático, con ello poder colaborar en la minimización de los efectos e impactos del cambio climático.

La cuenca hidrológica Aguas Zarcas ha presentado una baja en su caudal durante los último años debido al cambio climático, sin embargo ésta no ha afectado directamente a las actividades agropecuarias que rodean la zona, pero si ha afectado la generación hidroeléctrica de la comunidad.

Las actividades productivas se ubican en un sector que presenta un alto índice montañoso debido a sus cercanías con el Parque Nacional Juan Castro Blanco y en un área con un alto índice del recurso hídrico por su ubicación sobre las nacientes y fuentes de agua que nacen de la cuenca y el mismo parque, por lo que la vulnerabilidad a que se vean afectadas por el cambio climático no son tan altas como sucede en otras regiones del país; sin embargo eso no implica que no se deba prevenir y mitigar los efectos e impactos que se están comenzando a sentir en la zona de estudio.

Es importante reconocer la labor de organizaciones estatales y privadas que están tratando de informar y capacitar a algunos de los productores de la zona en la adaptación al cambio climático.

\section{Bibliografía, referencias y fuentes de información}

Abarca. K., (2015). Com.pers. Ingeniera Agrónoma Dos Pinos. Aguas Zarcas.

Abarca. K., (2015). Com.pers. Ingeniera Agrónoma Dos Pinos. Aguas Zarcas.

Ardila, A \& Vergara, W. (2011). El sector pecuario frente al cambio climático: una realidad incómoda. Recupera de: http://revistas.lasalle.edu.co/index.php/ca/article/ viewFile/1322/1207. Consultado el 24 de noviembre de 2015.

Arrieta. E., (2015). Com.pers. Productor lechero finca Arrieta. Aguas Zarcas.

Ávila. J. (2015). Trabajo de graduación: Percepción de los efectos e impacto del cambio climático en el desarrollo 
de las actividades productivas en la cuenca hidrográfica Aguas Zarcas en el distrito de Aguas Zarcas, San Carlos, Alajuela, Costa Rica (supervisado y dirigido por Ruiz. A.). Material inédito.

Barcelona Climate Change Talks. (2009). Politicas internacionales en la lucha contra el cambio climático. Recuperado de: http://www.bcn.cat/climatechange/ es/politiques-internacionals.html. Consultado el 26 de noviembre de 2015.

Camacho. E., (2015). Com.pers. Comerciante Pro Agro S.A. Aguas Zarcas.

Chacón. J., (2015). Com.pers. Productor Finca Avícola Bahía Baltry. Aguas Zarcas.

Comisión Europea. (2006). El cambio climático ¿Qué es?: Introducción para jóvenes. Recuperado de: http:// www.oei.es/decada/portadas/climate_change_youth_ es.pdf. Consultado el 21 de noviembre de 2015.

Dirección del Cambio Climático (DDC). (sf). ¿Cómo nos afecta?. Recuperado de: http://cambioclimaticocr.com/2012-05-22-19-44-14/como-nos-afecta. Consultado el 22 de noviembre de 2015.

Dirección del Cambio Climático (DDC). (sf). ¿Qué es el cambio climático?. Recuperado de: http://cambioclimaticocr.com/2012-05-22-19-44-14/que-es-elcambio-climatico. Consultado el 20 de noviembre de 2015 .

Dirección del Cambio Climático (DDC). (sf). Estrategia Nacional de Cambio Climático. Recuperado de: http://cambioclimaticocr.com/2012-05-22-19-42-06/ estrategia-nacional-de-cambio-climatico. Consultado el 26 de noviembre de 2015.

Dirección del Cambio Climático (DDC). (sf). Iniciativas. Recuperado de: http://cambioclimaticocr.com/201205-22-19-47-24/iniciativas. Consultado el 26 de noviembre de 2015 .

Echeverría, J. (2011). Evaluación de la vulnerabilidad futura del sistema hídrico al cambio climático. Recuperado de: http://www.preventionweb.net/files/28416_evaluaciondelavulnerabilidadfuturad.pdf. Consultado el 23 de noviembre de 2015.

Elbers, J. (2012). Desconocimiento y negación del cambio climático real. Recuperado de: http://www.cambioclimatico-bolivia.org/archivos/20120226005035_0.pdf. Consultado el 26 de noviembre de 2015.
Galante, A. (sf). Protocolo de Kioto y desarrollo sostenible. Recuperado de: http://rua.ua.es/dspace/ bitstream/10045/16032/1/cuadbiod34_03.pdf. Consultado el 26 de noviembre de 2015.

Galeano, M. (2004). Diseño de proyectos en la investigación cualitativa. Universidad Eafit. Recuperado de: https://books.google.co.cr/books?hl=es\&lr=\&id=u fsZQkjMUFEC\&oi=fnd\&pg=PA11\&dq=investiga cion + cualitativa + caracteristicas $\&$ ots $=4 \mathrm{CwuWv}-\mathrm{M}$ gf\&sig=unNZxzKpB7EXm3C46S38o0MSssI\&red ir_esc $=\mathrm{y} \# \mathrm{v}=$ onepage $\& \mathrm{q}=$ investigacion $\% 20$ cualitativa\%20caracteristicas $\& \mathrm{f}=$ false. Consultado el 30 de junio de 2015.

Gobierno de Costa Rica \& MINAE. (2015). Contribución prevista y determinada a nivel nacional de Costa Rica. Recuperado de: http://www4.unfccc.int/submissions/INDC/Published\%20Documents/Costa\%20 Rica/1/INDC\%20Costa\%20Rica\%20Version\%20 $2 \% 200 \% 20$ final\%20ES.pdf. Consultado el 22 de noviembre de 2015 .

Gonzales, J. (2008). Desarrollo sustentable, El cambio climático afecta a los negocios: Riesgos y oportunidades. Recuperado de: https://www.kpmg.com/MX/es/ IssuesAndInsights/ArticlesPublications/Documents/ DE2008/04CambioClimatico.pdf. Consultado el 26 de noviembre de 2015.

Granados, A. (2012). Aportes políticas de adaptación al cambio climático: "Impactos del cambio climático y la gestión del modelo alternativo de economía verde en América Central”. Recuperado de: http://library.fes.de/pdf-files/bueros/fesamcentral/09051.pdf. Consultado el 23 de noviembre de 2015.

Granados, A \& Madrigal, R. (2014). Posición del Estado de Costa Rica antes las conferencias de las partes de la Convención Marco de la Naciones Unidas sobre el cambio climático (COPs). Recuperado de: http://library.fes.de/pdf-files/bueros/fesamcentral/11115.pdf. Consultado el 26 de noviembre de 2015.

Grupo Intergubernamental de Expertos sobre el Cambio Climático. (2002). Cambio climático y biodiversidad: Documento técnico $V$ del IPCC. Recuperado de: https://www.ipcc.ch/pdf/technical-papers/climatechanges-biodiversity-sp.pdf. Consultado el 27 de noviembre de 2015 .

Jiménez, R. (2012). Costa Rica y el mundo ante el cambio climático: Enfoque y propuesta de política para 
enfrentar el cambio climático. Recuperado de: http:// www.ambientico.una.ac.cr/pdfs/ambientales/44.pdf. Consultado el 24 de noviembre de 2015.

Lobo. C., (2015). Com.pers. Productor finca lechera La Catarata. Aguas Zarcas.

MAG. (sf). Estado del arte en cambio climático, agricultura y seguridad alimentaria en Costa Rica. Recuperado de: http://www.mag.go.cr/bibliotecavirtual/a00327. pdf. Consultado el 25 de junio de 2015.

Ministerio de Medio Ambiente y Recursos Naturales Gobierno de El Salvador. (2013). Plan estratégico 2011-2020 y Metas de Aichi del Convenio sobre la Diversidad Biológica. Recuperado de: file://C:/ Users/270/Downloads/HI-02-BIO.pdf. Consultado el 26 de noviembre de 2015 .

Municipalidad de San Carlos. (2014). Plan de Desarrollo Distrital, Aguas Zarcas 2014-2024. Recuperado de: http://www.munisc.go.cr/ Documentos/NuestraMunicipalidad/Plan $\% 20 \mathrm{de} \% 20$ Desarrollo\%20Distrital\%20Aguas\%20Zarcas.pdf. Consultado el 24 de junio de 2015.

Ordaz, J \& ét. al. (2010). Costa Rica: Efectos del cambio climático sobre la agricultura. Recuperado de: http:// repositorio.cepal.org/bitstream/handle/11362/25921/ lcmex1972.pdf?sequence=1. Consultado el 23 de noviembre de 2015.

Organización de las Naciones para la Agricultura y la Alimentación. (sf). Mitigación del cambio climático y adaptación en la agricultura, la silvicultura y la pesca. Recuperado de: ftp://ftp.fao.org/docrep/fao/010/ i0142s/i0142S00.pdf. Consultado el 26 de noviembre de 2015 .

Organización de las Naciones Unidas para la Educación, la Ciencia y la Cultura. (2012). Educación para el desarrollo sostenible: Libro de consulta. Recuperado de: http://unesdoc.unesco.org/ images/0021/002167/216756s.pdf. Consultado el 26 de noviembre de 2015.

Pardo, M. (sf). El impacto social del Cambio Climático. Recuperado de: http://portal.uc3m.es/portal/page/portal/grupos_investigacion/sociologia_cambio_climatico/El\%20impacto\%20social\%20del\%20Cambio\%20 Clim\%E1tico_0.pdf. Consultado el 25 de junio de 2015 .
Parque Nacional del Agua. (2009). Parque Nacional del Agua Juan Castro Blanco. Recuperado de: http://www.parquenacionaldelagua.com/index. php?option=com_content $\&$ view=article $\&$ id=54 $\&$ Ite mid=83. Consultado el 24 de junio de 2015.

Pérez. J., (2015). Comp.pers. Licenciado y funcionario de la Agencia de Extensión Agropecuaria del MAG. Aguas Zarcas.

Rios, N \& Ibrahim, M. (2008). Impactos del cambio climático sobre los recursos hídricos. Recuperado de: http://orton.catie.ac.cr/repdoc/A5380E/A5380E.PDF. Consultado el 25 de junio de 2015.

Salas. C, (2015). Com.pers. Geógrafo y funcionario de la Municipalidad de San Carlos. Ciudad Quesada.

Solano, J \& Villalobos, R. (sf). Regiones y Subregiones Climáticas de Costa Rica: Instituto Meteorológico Nacional: Gestión de Desarrollo. Recuperado de: http://www.imn.ac.cr/publicaciones/estudios/Reg_ climaCR.pdf. Consultado el 25 de junio de 2015.

Soto. J., (2015). Com.pers. Productor finca acuícola Tilapias Soto. Aguas Zarcas.

Vergara \& ét. al. (2014). Agricultura y clima futuro en América Latina y el Caribe: Impactos sistémicos y posibles respuestas. Recuperado de: http://latinclima.org/sites/default/files/documentos/agricultura_y_ cambio_climatico_bid.pdf. Consultado el 23 de noviembre de 2015 .

Watson, R \& ét al. (1997). Resumen para responsables de políticas, Impactos regionales del cambio climático: evaluación de la vulnerabilidad. Recuperado de: https://www.ipcc.ch/pdf/special-reports/spm/regionsp.pdf. Consultado el 26 de noviembre de 2015.

World Vision. (sf). Manual de manejo de cuencas. Recuperado de: http://biblioteca.catie.ac.cr/cursocuencas/documentos/Manual_de_Manejo_de_ Cuencas_Vision_Mundial_mod.pdf. Consultado el 26 de noviembre de 2015.

Zumbado, F. (2011). Planes reguladores y su importancia para el sector agropecuario: Realidades del ordenamiento territorial. Recuperado de: http://www. kerwa.ucr.ac.cr/bitstream/handle/10669/11431/ Ordenamiento $\% 20$ Territorial $\% 20$ ecag 56. pdf?sequence=1\&isAllowed=y. Consultado el 26 de noviembre de 2015. 
